\title{
HIV-1 integrase inhibition: looking at cofactor interactions
}

\author{
"...the unique multimodal mode of action [of LEDGINs] and the lack of \\ cross-resistance with any known class of ARVs make them excellent candidates for \\ future CART, in particular for patients failing on current therapy."
}

\begin{abstract}
Keywords: drug discovery $\bullet$ HIV $\bullet$ host factors $\bullet$ integrase $\bullet$ LEDGF/p75 $\bullet$ protein-protein interaction
\end{abstract}

\section{HIV/AIDS: a threat to public health}

AIDS remains an extraordinary challenge for global public health. Since the beginning of the epidemic, almost 78 million people have been infected with HIV-1 and about 39 million people have died of HIV. According to WHO by the end of 2013 35 million people were living with HIV. By shifting from a deadly to a chronic disease HIV drug discovery has changed gears. Less research efforts focus on the development of novel classical antivirals and more efforts are undertaken to find a cure for the infection in order to reduce the current treatment burden for the patients. Interruption of combinatorial antiretroviral therapy (cART) typically results in a rebound of virus replication. This is primarily due to the fact that HIV ingeniously escapes from the continuous immune surveillance in reservoirs of latently infected cells that are not susceptible to drug therapy. Poor adherence and suboptimal treatment regimens, in particular of issue in vulnerable subpopulations of patients, together with rapid replication rates and the error-prone reverse transcription, accelerate the development and spread of resistant strains. As a consequence leaving the development of antiretrovirals out of sight might bear risks for disease management in the future. Refraining from classical drug development hence might come at a high cost in the long run. Hence there is a need to search for novel and better antiretrovirals to better control the pandemic and hopefully find ways to bring about a permanent remission of AIDS.

\section{Anti-HIV drug discovery}

Traditionally anti-HIV drugs target reverse transcription (nucleoside- NRTI, and nonnucleoside RT inhibitors NNRTI), proteolytic maturation (protease inhibitors PI [1]) or integrase strand transfer (integrase inhibitors INSTI [2]). Recently new strategies to inhibit HIV replication have come into the spotlight. Novel inhibitors targeting assembly and maturation through Gag have entered clinical trials (for a review on Gag drug discovery see [3]). Second, virushost interactions are believed to play a role in future disease control [4]. Targeting virus-host interactions introduces a novel approach into HIV therapy as these inhibitors by definition do not target directly the catalytic activities of the virus but prevent the interaction of the virus with host proteins. Until now this strategy remains experimental and proof-of-concept for the druggability of many virus-host interactions is still lacking, yet a generic proof-ofconcept has been provided by the discovery of LEDGINs targeting the interaction of HIV-1 integrase with the host factor LEDGF/p75 which are now in clinical development $[5,6]$. The term LEDGINs is generic for all small molecules binding to the well-defined LEDGF/p75 binding site on the HIV-1 dimer interface.

\section{The discovery of LEDGINs}

To accomplish productive infection, HIV needs to integrate its genome into the host chromatin inherently linking its fate to the host. Integration, catalyzed by the viral

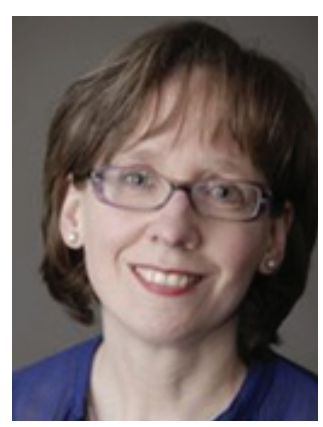

Frauke Christ

Author for correspondence: Laboratory for Molecular Virology and Gene Therapy Department of Pharmaceutical and Pharmacological Sciences, KU Leuven, Belgium frauke.christ@med.kuleuven.be

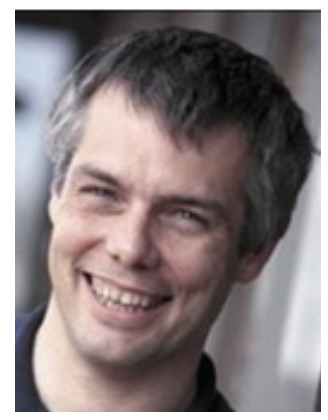

Zeger Debyser Laboratory for Molecular Virology and Gene Therapy Department of Pharmaceutical and Pharmacological Sciences, KU Leuven, Belgium 
protein integrase, therefore is a crucial step in the viral lifecycle. Integration into the preferred sites in the human genome can only be achieved through interaction with the cellular cofactor LEDGF/p75, tethering the proviral integration complex (PIC) to the chromatin [7]. LEDGF/p75 is a transcriptional coactivator and was first identified as an integrase cofactor through co-immunoprecipitation with HIV integrase [8]. Its crucial role in HIV replication was further evidenced via mutagenesis, RNAi-mediated depletion, transdominant overexpression of the INbinding domain (IBD) of LEDGF/p75 and cellular knock out studies [9-16]. LEDGF/p75 is encoded by the PSIP1 gene, which produces two proteins through alternative splicing (p52 and p75), both sharing the $\mathrm{N}$-terminal region responsible for nuclear localization (classical NLS) and chromatin binding (PWWP domain). Yet p75 contains a C-terminal protein-binding domain, integrase binding domain (IBD), which interacts with cellular and viral proteins linking this protein to different diseases such as HIV/AIDS and malignancies [7,17-20]. The IBD of LEDGF/p75 tightly associates with the core domain of HIV integrase [21]. Through its chromatin interaction LEDGF/p75 therefore tethers IN to the chromatin of the cell, facilitating integration and determining integration site selection [14,22-23].

In 2005 the cocrystal structure of LEDGF-IBD and HIV-1 IN-catalytic core domain (CCD) was reported [24], identifying crucial amino acids residues of LEDGF/p75 mediating the interaction with IN (Ile365, Asp366, Phe406, Val408). Two regions of the IN catalytic core domain dimer are in direct contact with LEDGF/p75, namely the region around Trp131 and Trp132 and the region extending from Ile161 to Glu170. The protein-protein interaction surface of LEDGF/p75 and IN hence is based on a well-defined pocket, limited in size and composed of multiple hydrophobic and hydrogen bond interactions. The described characteristics indicated that disruption of the interaction by small molecules is feasible and prompted us to initiate drug discovery against this target. Two elegant technologies validated that the LEDGF/p75-IN interaction is indeed a druggable target for HIV-replication. First depletion of LEDGF/p75 through RNAi or knock-out potently inhibited HIV-1 replication in cell culture [12,14-16]. Secondly HIV-1 replication was potently blocked in cells over-expressing IBD transdominantly [11]. By serial passaging of HIV in cells overexpressing this LEDGF/p75 fragment, a virus strain resistant to the phenotype was selected. Interestingly two IN mutations were required for resistance, A128T and E170G, confirming the mutagenesis data, the validity of the
IBD/IN catalytic core domain structure and more importantly the likely amenability for small molecule inhibitors disrupting the interaction [25]. Further support was provided by the report of small peptides interfering with the interaction [26].

Validation of the LEDGF/p75-IN interaction and its potential druggability has triggered several drug discovery programs for the identification of small-molecule protein-protein interaction inhibitors (SMPPIIs). These include high throughput screening (HTS) of diverse chemical libraries, in silico screening of virtual compound libraries and structure-based design of de novo small molecules on the basis of a pharmacophore model [5].

In 2010, we reported the first LEDGF/p75-IN SMPPIIs with antiviral activity in cell culture, all derivatives of 2-(tert-butoxy)-2-substituted acetic acid [27]. The discovery of this class of compounds was initiated by the construction of a consensus pharmacophore representing common steric and electronic features identified on different available crystal structures of the CCD of IN which were predicted to be critical for LEDGF/p75 binding in the hydrophobic pocket at the IN dimer interface. Around 200,000 commercially available diverse compounds were filtered and fitted to the pharmacophore model in a virtual screen. Iterative rounds of structural biology, medicinal chemistry optimization and biological evaluation led to the discovery of the first compounds with antiviral activity. Further optimization led to the discovery of CX014442, a compound with modest cellular toxicity, potent antiviral efficacy in the low nanomolar range resulting in a significant selectivity in cell culture $\left(\mathrm{EC}_{50 \text { ІІІ }}=69 \pm\right.$ $3 \mathrm{nM}$ and high selectivity, SI $=1391$ ) and allowing full biological profiling of this novel mechanistic class of anti-HIV compounds [28]. Whereas our program was based on structure-based design, Boehringer Ingelheim discovered similar compounds through a HTS approach aimed at identifying inhibitors of integration beyond the strand transfer step, demonstrating that drug discovery programs can become convergent even when starting from different initial screening approaches.

Optimizing the potency of LEDGINs allowed for detailed mechanistic studies, which revealed that all described and characterized molecules of this class of small molecules share a common multiplex mechanism of action (MOA) which is not found in any other class of anti-HIV compounds and to our knowledge neither in other infectious disease drugs. In stark contrast to INSTIs, LEDGINs inhibit both catalytic activities of integrase, the $3^{\prime}$ processing and strand transfer reaction. Unlike INSTIs, which require the presence of the HIV intasome (integrase 
assembled on $3^{\prime}$ processed long terminal repeats of the viral genome), LEDGINs only reach full efficacy if present before intasome assembly [28-30]. Careful analysis of the inhibition of the catalytic activity of integrase demonstrated that LEDGINs stabilize the assembly of integrase oligomers and therefore interfere with the formation of a catalyitically competent intasome. Thus LEDGINs are genuine allosteric integrase inhibitors and first of their kind. LEDGINs have a dual mode of action inhibiting the interaction of integrase with the cellular cofactor LEDGF/p75 and therefore reducing the efficacy of integration and inhibiting the catalytic activity through a novel allosteric site. Much attention has been brought to the discussion whether one or the other is contributing most to the antiviral activity of LEDGINs. Although this discussion is of interest from a mechanistic point of view, one should keep in mind that in the patient the cellular cofactor LEDGF/p75 is indispensable and therefore both activities cannot be separated from each other in the real life situation.

As expected from allosteric inhibitors LEDGINs are not cross-resistant with INSTIs and vice versa. Of major importance is the finding that combination of both classes of integrase inhibitors leads to additive effects in cell culture paving the way toward combination therapy, the gold standard in HIV treatment. The sound activity against all clades of HIV as well as viruses resistant against other classes of antivirals provides further confidence in the clinical value of LEDGINs. So far, one LEDGIN developed by Boehringer Ingelheim demonstrated excellent pharmacokinetic profile in rat and dog and advanced into a Phase I clinical trial [6].

While studying the detailed MOA of LEDGINs, it came as a surprise that LEDGINs not only inhibit early replication, but also late replication steps. Virions produced in the presence of LEDGINs exert severe defects in reverse transcription, nuclear import and integration in the following round of replication. Detailed analysis by electron microscopy of virions produced in the presence of LEDGINs evidenced severe morphological defects in virions. Yet proteolytic processing and genomic RNA processing were not defective. This effect is directly linked to the binding of LEDGINs in the LEDGF/p75 binding pocket and the stabilization of multimerization of integrase. This impairment of infectivity of the progeny virus is unique for LEDGINs and is not part of the mechanism of other integration inhibitors such as raltegravir or other classes of early replication inhibitors, such as entry blockers, NRTIs (nucleoside reverse transcriptase inhibitors) or NNRTIs (non-nucleoside reverse transcriptase inhibitors). The finding that LEDGINs not only block the integration of the viral particle but additionally impair the infectivity of viral particles, when present during production, makes them genuine and highly interesting candidates for further clinical development. On the other side, this finding identifies a to date unknown function of HIV-1 integrase in maturation of the viral particle. Discovery of new inhibitors with novel MOA thus draws the bow back basic research and contributes to a better understanding of the disease biology of infectious diseases. LEDGIN's destiny will not be solely depending on the antiviral activity but more importantly on pharmacokinetics/pharmacodynamics and tolerability. The development of LEDGINs for clinical use is in its early days and proof-of-concept in Phase II clinical trials is still awaited for. However, the unique multimodal mode of action and the lack of cross-resistance with any known class of ARVs make them excellent candidates for future cART, in particular for patients failing on current therapy.

\section{Conclusion}

In the past decade the identification and validation of LEDGF/p75 as an authentic cofactor of HIV has been one of the most exciting research lines in HIV biology. The discovery of LEDGINs has been recognized, both inside the HIV field but also by a broader scientific public, as a showcase of (academic) drug discovery. Host interactions are crucial for all human pathogens. Therefore LEDGINs might encourage further drug discovery efforts and might pave the way toward the discovery of new targets and SMPPIIs with novel mechanisms of action. In case the novel drug targets the host instead of the viral protein, resistance is unlikely to develop making pathogen-host interaction even more interesting drug targets. This is of utmost importance in particular in light of pathogens with high occurrence of resistance such as multiple resistant Staphylococcus aureus (MRSA) in hospital settings. Crucial is solid target validation as described here for the LEDGF/p75-IN interaction prior to the start of drug discovery.

\section{Financial \& competing interests disclosure}

The authors have no relevant affiliations or financial involvement with any organization or entity with a financial interest in or financial conflict with the subject matter or materials discussed in the manuscript. This includes employment, consultancies, honoraria, stock ownership or options, expert testimony, grants or patents received or pending, or royalties.

No writing assistance was utilized in the production of this manuscript. 


\section{References}

1 Pokorna J, Machala L, Rezacova P, Konvalinka J. Current and novel inhibitors of HIV protease. Viruses 1(3), 1209-1239 (2009).

2 Mesplede T, Wainberg MA. Integrase strand transfer inhibitors in HIV therapy. Infect. Dis. Ther. 2(2), 83-93 (2013).

3 Spearman P. HIV-1 Gag as an antiviral target: development of assembly and maturation inhibitors. Curr. Top. Med. Chem. (2015) (Epub ahead of print).

4 Weydert C, De Rijck J, Christ F, Debyser Z. Targeting virus-host interactions of HIV replication. Curr. Top. Med. Chem. (2015) (Epub ahead of print).

5 Christ F, Debyser Z. The LEDGF/p75 integrase interaction, a novel target for anti-HIV therapy. Virology 435(1), 102-109 (2013).

6 Fenwick C, Amad M, Bailey MD et al. Preclinical profile of BI 224436, a novel HIV-1 non-catalytic-site integrase inhibitor. Antimicrob. Agents Chemother. 58(6), 3233-3244 (2014).

7 Debyser Z, Christ F, De Rijck J, Gijsbers R. Host factors for retroviral integration site selection. Trends Biochem. Sci. 40(2), 108-116 (2015).

8 Cherepanov P, Maertens G, Proost P et al. HIV-1 integrase forms stable tetramers and associates with LEDGF/p75 protein in human cells. J. Biol. Chem. 278(1), 372-381 (2003).

9 Ciuffi A, Bushman FD. Retroviral DNA integration: HIV and the role of LEDGF/p75. Trends Genet. 22(7), 388-395 (2006).

10 Ciuffi A, Llano M, Poeschla E et al. A role for LEDGF/ p75 in targeting HIV DNA integration. Nat. Med. 11(12), 1287-1289 (2005).

11 De Rijck J, Vandekerckhove L, Gijsbers R et al. Overexpression of the lens epithelium-derived growth factor/p75 integrase binding domain inhibits human immunodeficiency virus replication. J. Virol. 80 (23), 11498-11509 (2006).

12 Llano M, Saenz DT, Meehan A et al. An essential role for LEDGF/p75 in HIV integration. Science 314(5798), 461-464 (2006).

13 Llano M, Vanegas M, Hutchins N, Thompson D, Delgado $S$, Poeschla EM. Identification and characterization of the chromatin-binding domains of the HIV-1 integrase interactor LEDGF/p75. J. Mol. Biol. 360(4), 760-773 (2006).

14 Schrijvers R, De Rijck J, Demeulemeester J et al. LEDGF/ p75-independent HIV-1 replication demonstrates a role for HRP-2 and remains sensitive to inhibition by LEDGINs. PLoS Pathog. 8(3), e1002558 (2012).

15 Shun MC, Raghavendra NK, Vandegraaff N et al. LEDGF/ p75 functions downstream from preintegration complex formation to effect gene-specific HIV-1 integration. Genes Dev. 21(14), 1767-1778 (2007).

16 Vandekerckhove L, Christ F, Van Maele B et al. Transient and stable knockdown of the integrase cofactor LEDGF/ p75 reveals its role in the replication cycle of human immunodeficiency virus. J. Virol. 80(4), 1886-1896 (2006).

17 Bartholomeeusen K, Christ F, Hendrix J et al. Lens epithelium-derived growth factor/p75 interacts with the transposase-derived DDE domain of PogZ. J. Biol. Chem. 284(17), 11467-11477 (2009).

18 Bartholomeeusen K, De Rijck J, Busschots K et al. Differential interaction of HIV-1 integrase and JPO2 with the C terminus of LEDGF/p75. J. Mol. Biol. 372(2), 407-421 (2007).

19 Maertens GN, Cherepanov P, Engelman A. Transcriptional co-activator $\mathrm{p} 75$ binds and tethers the Myc-interacting protein JPO2 to chromatin. J. Cell Sci. 119(Pt 12), 2563-2571 (2006).

20 Yokoyama A, Cleary ML. Menin critically links MLL proteins with LEDGF on cancer-associated target genes. Cancer Cell 14(1), 36-46 (2008).

21 Maertens G, Cherepanov P, Pluymers W et al. LEDGF/ p75 is essential for nuclear and chromosomal targeting of HIV-1 integrase in human cells. J. Biol. Chem. 278(35), 33528-33539 (2003).

22 Ciuffi A, Mitchell RS, Hoffmann C et al. Integration site selection by HIV-based vectors in dividing and growtharrested IMR-90 lung fibroblasts. Mol. Ther. 13(2), 366-373 (2006).

23 Shun MC, Botbol Y, Li X et al. Identification and characterization of PWWP domain residues critical for LEDGF/p75 chromatin binding and human immunodeficiency virus type 1 infectivity. J. Virol. 82(23), 11555-11567 (2008).

24 Cherepanov P, Sun ZY, Rahman S, Maertens G, Wagner G, Engelman A. Solution structure of the HIV-1 integrasebinding domain in LEDGF/p75. Nat. Struct. Mol. Biol. 12(6), 526-532 (2005).

25 Hombrouck A, De Rijck J, Hendrix J et al. Virus evolution reveals an exclusive role for LEDGF/p75 in chromosomal tethering of HIV. PLoS Pathog. 3(3), e47 (2007).

26 Al-Mawsawi LQ, Christ F, Dayam R, Debyser Z, Neamati N. Inhibitory profile of a LEDGF/p75 peptide against HIV-1 integrase: insight into integrase-DNA complex formation and catalysis. FEBS Lett. 582(10), 1425-1430 (2008).

27 Christ F, Voet A, Marchand A et al. Rational design of smallmolecule inhibitors of the LEDGF/p75-integrase interaction and HIV replication. Nat. Chem. Biol. 6(6), 442-448 (2010).

28 Christ F, Shaw S, Demeulemeester J et al. Small-molecule inhibitors of the LEDGF/p75 binding site of integrase block HIV replication and modulate integrase multimerization. Antimicrob. Agents Chemother. 56(8), 4365-4374 (2012).

29 Kessl JJ, Jena N, Koh Y et al. Multimode, cooperative mechanism of action of allosteric HIV-1 integrase inhibitors. J. Biol. Chem. 287(20), 16801-16811 (2012).

30 Tesina P, Cermakova K, Horejsi M et al. Multiple cellular proteins interact with LEDGF/p75 through a conserved unstructured consensus motif. Nat. Commun. 6, 7968 (2015). 